\title{
Reconnecting the brain after injury
}

During the first few hours of acute brain injury, such as a blow to the head or a stroke, brain cells are damaged and permanently lost, resulting in loss of functionality. Current treatment options for such brain injuries are limited and are only effective if administered during the first few hours after the insult. Now, Abe et al. have found that a small compound, edonerpic maleate, enhances synaptic plasticity and functional recovery in later phases after a traumatic injury to the brain.

Although the number of neurons in the adult brain is constant, the connections among them are not. Brain circuits have synaptic plasticity, building and eliminating synapses and adapting the strength of existing connections. The team led by Takuya Takahashi had previously reported that delivery of synaptic glutamate $\alpha$-amino-3-hydroxy-5methyl-4-isoxazole propionic acid receptor (AMPAR) to the synapse mediates functional cortical reorganization by enhancing intact sensory systems that compensate for visual loss. "This finding drove me to think of a small molecule that facilitates synaptic AMPAR delivery, and which could accelerate recovery after brain damage with rehabilitative training," explains Takahashi.

That molecule is edonerpic maleate, which protects and modifies the morphology of neurons. Although the safety of this compound has been proven in a phase I clinical trial, its clinical application remains to be determined. To explore the effect of edonerpic maleate on neuronal function, the authors focused on experience-dependent synaptic plasticity. Oral administration of the compound twice a day to adult mice with and without whiskers (to assess experience-related input) for 3 weeks resulted in facilitation of synaptic AMPAR delivery to the barrel cortex in the presence of whiskers. Further experiments showed that edonerpic maleate binds directly to collapsin response mediator protein 2 (CRMP2) - a protein involved in synaptic plasticity and learning - and regulates its multimerization. In turn, CRMP2 activates actin-depolymerizing factor (ADF)/cofilin, which mediates AMPAR trafficking during synaptic plasticity.

The authors showed that edonerpic maleate administered 1 day after severe motor cortical cryoinjury could accelerate the effect of rehabilitation on motor function recovery in mice trained to reach for food pellets. This effect was mediated by CRMP2 as CRMP2-deficient mice were not able to recover the reaching task performance.

These results were confirmed in an internal capsule haemorrhage model in macaque monkeys trained in two tasks (reach-and-grab task and vertical-slit task). In this case, edonerpic maleate was administered during the recovery phase days after the insult (as soon as the monkeys showed signs of motor function) and not during the few hours in the acute phase of brain injury. Edonerpic maleate administration enhanced the recovery of both the success rate and the time to retrieval in both tasks. In

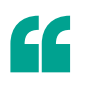

a small

compound, edonerpic

maleate,

enhances

synaptic

plasticity and

functional

recovery in

later phases

after a

traumatic

injury to

the brain addition, whereas the monkeys that received treatment showed dexterous hand movements, including precision grip, this move was rarely observed in the untreated monkeys.

"The most important step will be to prove this effect in stroke patients with a clinical trial and we are currently preparing for it," says Takahashi. "Another important step will be to prove the effect of this compound in other diseases such as spinal cord injury. Most spinal cord injuries result from partial tears and the descending pathway should still remain intact. Thus, functional cortical reorganization can compensate for the lost motor function."

Because edonerpic maleate has been assessed in clinical trials (phase II for Alzehimer disease) and its safety has already been proved, this compound could offer a promising treatment option for brain damage.

\section{Teresa Villanueva}

ORIGINAL ARTICLE Abe, H. et al. CRMP2-binding compound, edonerpic maleate, accelerates motor function recovery from brain damage. Science 360, 50-57 (2018) 